\title{
COVID-19: The Healthcare Preparedness of healthcare delivery systems in Public Health emergencies
}

\section{Surekha Kishore ${ }^{1}$, Sheen Job ${ }^{2}$}

${ }^{1}$ Professor \& Head, Department of Community \& Family Medicine, All India Institute of Medical Sciences, Rishikesh, Uttarakhand; ${ }^{2}$ MPH PG, Department of Community \& Family Medicine, All India Institute of Medical Sciences, Rishikesh, Uttarakhand

\begin{tabular}{|c|c|c|c|c|c|c|c|}
\hline Abstract & Introduction & Methodology & Results & Conclusion & References & Citation & Tables/ Figures \\
\hline
\end{tabular}

\section{Corresponding Author}

Dr Surekha Kishore, Professor \& Head, Department of Community \& Family Medicine, All India Institute of Medical Sciences, Rishikesh, Uttarakhand.

E Mail ID: surekhakishore@rediffmail.com

\section{Citation}

Kishore S, Job S. COVID-19: The Healthcare Preparedness of healthcare delivery systems in Public Health emergencies. Indian J Comm Health. 2020;32(1):01-03.

Source of Funding: Nil Conflict of Interest: None declared

\section{Article Cycle}

Received: 04/03/2020; Revision: 08/03/2020; Accepted: 12/03/2020; Published: 31/03/2020

This work is licensed under a Creative Commons Attribution 4.0 International License.

\section{Background}

There is a public health alert in the country since the news of the Coronavirus outbreak. Though not very alarming, as only 126 cases have been confirmed so far, who are largely foreign tourists from outside India, Indians traveling abroad. The entire government health administration at various levels are constantly monitoring the situation. The precarious situation is being closely monitored by the Ministry of Health and Family Welfare. The Joint Monitoring Group (JMG) convened under the Chairmanship of Director General of Health Services (DGHS) with representations from various stakeholders (health and non-health sectors) and WHO (1) are instrumental in bringing about the responsiveness in the health systems.

Seventy years on, since the founding of the People's Republic of China (2), the country has made tremendous strides in Health care, up until now, the novel Coronavirus 2019 has wrecked the health fabric of the part of People's Republic of China with its ravaging onslaught.

However, the incident and contingency measures undertaken on a warfooting after the identification of the Viral pneumonia (as a novel strain among the group of Coronaviruses) gives a promise for the Nation, struggling to contain the epidemic. China, has the largest population in the World, and so also is their vast representation in the entire globe. It is heartening to see however, that the disease outbreak is slowly edging towards pandemic proportions. The burden of relentless and painstaking care round the clock by the frontline health workers is commendable and is indeed a lesson to imbibe for the whole world. The fear and apprehension for the disease has lead to relocation and migration of large sections of the population of Wuhan city, China. The powerful leadership of China and its policies on Health are indeed robust, the advantage of pooling resources and the technological support coupled with the experience of containing the SARS (severe acute respiratory syndrome) epidemic in 2003 in Guangdong China. (3)

The Director-General WHO Dr Tedros Ghebreyesus, declared COVID-19 a global pandemic on 11 ${ }^{\text {th }}$ March 2020. There are enormous gaps in the understanding of the dynamics of the disease. Very little is known about the origin of the new strain of the virus, if the media reports are to be believed, it is a controversial story. There was a false hope, in later part of the previous century, that the infectious epidemics have been curbed. In time, we witnessed a spate of emerging infectious illnesses of viral origin spilling over from wildlife reservoirs. Striking examples either directly or via domestic animals, were AIDS from chimpanzees, influenza from wild birds, Ebola, SARS and MERS from bats, and Dengue, Chikungunya and Zika from mosquitoes. This paved the way for the unprecedented spread of infections in humans and animals with dramatic consequences for public and animal health, animal welfare, food supply, economies, and biodiversity. (4) Emergence of epidemics such as novel Coronavirus, in a setting shrouded in mystery, the Chinese govt tight lipped about the outbreak also casts doubts on the subject of Biowarfare agents used in terror strikes or in military strategies 


\section{Health Systems preparedness}

The core capacities are being strengthened in India for timely detection. On 30 Jan 2020, the World Health Organization declared COVID-19 as Public Health Emergency of International Concern. However, as a matter of abundant precaution the Ministry of Health has instructed screening of International travellers from China at designated airports namely, Delhi, Mumbai and Kolkata through thermal scanners. In coordination with Ministry of Civil Aviation, in-flight announcements are also being made. A travel advisory has also been issued to the passengers travelling to and from China on the issue. A copy of the same is also available on the Health Ministry website.

The measures of preparedness for combating the outbreak includes laboratory diagnosis (Figure 1), surveillance, infection prevention and control (IPC) and risk communication. Integrated Disease Surveillance Program (IDSP) is geared up for community surveillance and contact tracing. The NIV Pune, ICMR Laboratory is coordinating the testing of samples for $\mathrm{nCoV}$ in the country. The hospital preparedness with regard to management and infection prevention control facilities has been also reviewed during high level meetings and advisories, and IPC Guidelines have been shared with the States. Adequate stocks of logistics including PPE is available. State governments have also been advised on the necessary precaution to be taken up on the issue. Ministry of Health is also in touch with Ministry of External Affairs, and the immigration officers at the said airports have been sensitized.

\section{Salient features}

During any public health emergency, it is the sharing of vital information of disease, its source, various other determinants that is of paramount importance. This data sharing provides great insights for formulating Policies and instituting Strategies on the subject of diseases caused by the novel strains of emerging new viruses. Epidemiological parameters are usually obtained from a consecutive timeline of the number of reported cases and contact-tracing data. (4)

To understand the disease, the basic epidemiological triad has to be well understood. The host, the agent and the environment. Its from the Coronaviridae family, a single stranded RNA with virions appearing like a solar corona. "From genetic sequencing data, it appears that there was a single introduction into humans followed by human-tohuman spread. This novel virus shares $79.5 \%$ of genetic sequence with SARS-CoV and has $96.2 \%$ homology to a bat coronavirus.

In addition, 2019-nCoV shares the same cell entry receptor" adds the researcher from University of Michigan. (4) The incubation period of this virus is between 5-14 days. Eating exotic mammals is a culture in south China and hence the possibility of re-emergence of novel viruses from animals and laboratories is a strong possibility. Transmission of the disease is still not very defined as cases are reported also in asymptomatic phase. (5) Much has to be understood, as the gaps in the disease dynamics is an enigma.

From the repository of data available with the Wuhan General Hospital, Coronaviruses cause colds with major symptoms, such as fever and sore throat, primarily in the winter and early spring seasons. Coronaviruses can cause pneumonia - either direct viral pneumonia or a secondary bacterial pneumonia - and may cause bronchitis - either direct viral bronchitis or a secondary bacterial bronchitis. Death is caused by severe respiratory failure.

The latest WHO bulletin on the sitrep issued on Feb 13 2020 says 46997 confirmed cases with 1369 deaths. In India, three cases have been confirmed and are underging treatment in southern state of Kerala. The observations as published in the Lancet Journal January 2020, depicted in Table 1 shows a comparison of the epidemics caused by the Corona viruses and its characteristics. The (Figure 1) however, shows the timeline of the early stages of the outbreak of the 2019

\section{Preventive approaches}

In response to the outbreak, Chinese authorities suspended travel in and out of Wuhan. Similar travel bans followed in other cities in Hubei Province, and in total close to 50 million people have been quarantined, an unprecedented effort to control any infectious disease. Travel embargo in and out of China and flight screening at airports were introduced. The WHO declared the outbreak a Public Health Emergency of International Concern. The quarantine measures for control is uncertain as it has not been found effective in similar outbreaks and pandemics on earlier occasions. It is a daunting task to find the clinical control measures as no vaccines or effective antivirals are reported for use. Such public health emergencies respect no borders or political boundaries.

The basic public health measures such as staying home when ill, handwashing, and respiratory etiquette including covering the mouth and nose during sneezing and coughing were effective in controlling SARS. The epidemic is a challenge on its own, with its enormity of numbers and frontline health workers who are the most susceptible. The intersectoral coordination between all stakeholders imparts the experience and education to themselves and the public at large. (5)

The recommendations on the protective masks by the National Health commission, has indeed provided further insights to the preventive practices in acquiring droplet infection in Coronavirus (nCoV2019)

\section{Policy \& Strategy for Emerging infectious outbreaks}

The purpose and scope of the IHR (International Health regulations), are "to prevent, protect against, control and provide a public health response to the international 
spread of disease in ways that are commensurate with and restricted to public health risks, and which avoid unnecessary interference with international traffic and trade." With travel and migration increasing over the decade more measures for screening of illnesses may have to be made available at entry ports and points. (6) A central and historic responsibility for the World Health Organization (WHO) has been the management of the global regime for the control of the international spread of disease.

Under Articles 21(a) and 22, the Constitution of WHO confers upon the World Health Assembly the authority to adopt regulations "designed to prevent the international spread of disease" which, after adoption by the Health Assembly, enter into force for all WHO Member States that do not affirmatively opt out of them within a specified time period (6). National capacities to detect, report, and respond are the foundation for preparedness.

Policy makers have a daunting task of formulating strategies and policy prescriptions to tackle zoonotic diseases of pandemic proportions. Many communities in various cultures follow the tradition of consuming exotic animal delicacies which is now a potential threat looming large globally. Though the understanding about the cultural practises and disease is lacking, more investigation and research can ascertain causes of the animal to human transmission in zoonotic illness. (7)

\section{Conclusion}

The novel Coronavirus 2019 has taken the world by storm. It is time to reflect upon the emerging viruses which lead to significant morbidity and are potentially fatal. The SARS, Ebola, NIPAH viruses have proven beyond doubt that the zoonotic diseases have the capability to wipe out sections of the population in time if the spread is not stemmed with prompt preventive and control measures. Early identification and strict surveillance are essential for containment of the emerging diseases from crossing over regional boundaries and cause harm globally. Policy makers have a task at hand to bring about a change in the scenario and the resultant morbidity in the recent outbreaks, which is in enormous numbers. Sharing of information and essential data that are crucial for preparedness in combating epidemics across regional borders need to be emphasized in International protocol. A committed effort by the frontline workers can bring about a rapid change in the progression of the outbreak. Moreover, it should be remembered that selfless service by the frontline workers, without rest or recuperation leads to severe burn out and exhaustion due to work stress. Furthermore, research and data needs to be collected on diseases contracted from the given geographical regions where there is a tradition of eating exotic animal delicacies, and incidentally are also the places of origin of virulent epidemics. These insights can bring about a strategic change in drafting appropriate policies for dissemination to all stakeholders, through the International Health Regulations or suitable channels. Finally, all Countries must join hands to curb this epidemic with improvised approaches, of prompt data sharing, documentation and good political commitment.

\section{References}

1. https://pib.gov.in/PressReleaselframePage.aspx?PRID=1599665

2. Phelan AL, Katz R, Gostin LO. The Novel Coronavirus Originating in Wuhan, China. Jama. 2020;2019-20.

3. Merianos A, Peiris M. International Health Regulations (2005). Lancet. 2005;366(9493):1249-51.

4. C. DR, P.N. M. 2019 Novel Coronavirus-Important Information for Clinicians. JAMA [Internet]. 2020; Available from: http://www.embase.com/search/results?subaction=viewrecord\&f rom=export\&id $=\mathrm{L} 630831394$

5. Liu P, Shi L, Zhang W, He J, Liu C, Zhao C, Kong SK, Loo JF, Gu D, Hu $L$ (Nov "Prevalence and genetic diversity analysis of human coronaviruses among cross-border children". Virology Journal. 14 (1): 230. doi:10.1186/s12985-017-0896-0. PMC 5700739. PMID 29166910.

6. Zhang C, Wang M. Origin time and epidemic dynamics of the 2019 novel coronavirus. bioRxiv [Internet]. 2020;2020.01.25.919688. Available from:

https://www.biorxiv.org/content/10.1101/2020.01.25.919688v2

7. National Health Commission People's Republic of China, website accessed on 14 Feb 2020 www.en.nhc.gov.cn

\section{Figures}

\section{FIGURE 1 DESIGNATED LABS IN INDIA FOR NCOV-19 TESTING}

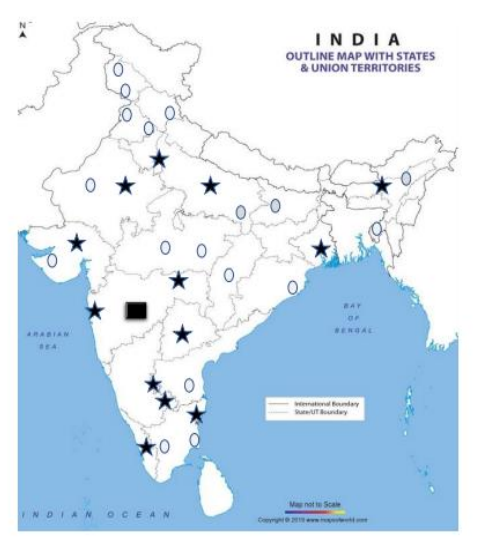

